\title{
The effect of positive end-expiratory pressure during anesthesia on arterial oxygen saturation after surgery in patient undergoing cesarean section
}

\author{
Mahmood Ganjifard (1), Masoumeh Samii (2), Samaneh Kouzegaran (3), Amir \\ Sabertanha (1)
}

(1) Department of Anesthesia, Emam Reza General Hospital, Birjand University of Medical Sciences, Birjand, Iran; (2) Student of Anesthesia, Emam Reza General Hospital, Birjand University of Medical Sciences, Birjand, Ira; (3) Department of Pediatrics, Valiasr General Hospital, Birjand University of Medical Sciences, Birjand, Iran.

This article is distributed under the terms of the Creative Commons Attribution Noncommercial License (CC BY-NC 4.0) which permits any noncommercial use, distribution, and reproduction in any medium, provided the original author $(s)$ and source are credited.

\begin{abstract}
One of the major complications of general anesthesia in the recovery room is arterial oxygen desaturation and hypoxemia. Positive end-expiratory pressure (PEEP) can improve arterial oxygen saturation by increasing FRC. This study aims to evaluate the effects of applying PEEP on arterial oxygen saturation and hemodynamic parameters in the patient undergoing cesarean section in VALIASR hospital. In this double blind clinical trial we randomly allocated 120 patients of class 1 and 2 ASA scheduled to undergoing cesarean section into 3 group (in 40).Different levels of PEEP $(0,5$ and $10 \mathrm{CmH} 2 \mathrm{o})$ were applied to each group while zero PEEP was established as control. All other variables (anesthesia and surgery techniques) were the same for all patients SPO2, noninvasive mean arterial pressure and heart rate were measured before, during and after surgery (Recovery room). The comparison of noninvasive arterial blood pressure and heart rate during and after surgery did not show significant differences but mean 02 saturation in group B ( $5 \mathrm{cmH} 2$ o PEEP) and $\mathrm{C}(10 \mathrm{~cm} \mathrm{H} 2$ o PEEP) in PACU was higher than control group ( $98.30 \pm 0.93$ and $98.50 \pm 0.90$ as opposed to97.12 \pm 1.15 respectively) $\mathrm{P}<0.001$. In light of results applying PEEP is effective in preventing desaturation after surgery and improving respiratory indexes without the significant hemodynamic changes, the result of using five $\mathrm{cmH} 2 \mathrm{O}$ PEEP is more efficient and satisfying.
\end{abstract}

Key Words: positive end-expiratory pressure, arterial oxygen saturation, cesarean section

Eur J Transl Myol 28 (1): 93-97, 2018

In cesarean, most patients tend to have general anesthesia instead of other modalities. It is thus vital to prevent complications occurring after general anesthesia and mechanical ventilation intubation. ${ }^{1,2}$ Although the use of mechanical ventilation in some cases causes pulmonary damage, this method is essential for the survival of patients undergoing surgery. When associated with atelectasis and hypoxia, these damages could undermain the respiratory process., ${ }^{3,4}$ After general anesthesia and after physiological removal of the intubation, the functional remaining capacity (FRC) is eliminated, eventually causing damage of the alveoli. One of the postoperative side effects that may occur is severe decrement of oxygen saturation of blood, i.e., hypoxia. Various effects of postoperative hypoxia could be right-to-left lung intraventricular shunts, atelectasis, FRC reduction, congestive heart failure, hypotension (due to residual effects of anesthetic drugs), disseminated hypoxia (due to $\mathrm{N}_{2} \mathrm{O}$ deficiency), pulmonary embolism, pneumothorax, postoperative shivering, sepsis, blood transfusion-induced lung damage. High age, obesity and aspiration of the contents of the stomach are some of the cofactors. Among these complications, atelectasis and alveolar hypoventilation are seen frequently after general anesthesia. ${ }^{5,6}$ Pulmonary atelectasis is the main event that causes abnormalities in gas exchange and hypoxia during anesthesia. It was suggested that the primary treatment of collapse in patients undergoing anesthesia is to exert positive pressure at the end of the exhalation (PEEP). Also, using the different levels of positive end-expiratory pressure is suggested for treating of atelectasis and improving arterial oxygenation. Pulmonary gas exchange is usually affected and reduced during general anesthesia. Also, the FRC decreased about $21 \%$ during induction of anesthesia. Several reports are available on the effects of 
Table 1. Comparison of mean age and weight in patients in the three groups

Variables

Age

Weight
Mean +/- SD

$\mathbf{A}$

$29 / 13 \pm 4 / 65$

$67 / 00 \pm 9 / 71$

\section{B}

$27 / 60 \pm 4 / 27$

$68 / 25 \pm 8 / 31$

\section{C}

$26 / 90 \pm 4 / 88$

$67 / 35 \pm 6 / 42$
Results

\begin{tabular}{ll}
\hline $\mathbf{p}$ & $\mathbf{F}$ \\
$0 / 09$ & $2 / 44$ \\
$0 / 7$ & $0 / 24$
\end{tabular}

using the different levels of PEEP on ventilation over the FRC of patients during the general anesthetic. Reduction of FRC could be partially restored through reconditioning by using PEEP. ${ }^{7}$ On the other hand, it has been proven that PEEP reduces cardiac input and ioutput, the diastolic volume of left ventricue, central arterial pressure and central venous pressure, inducing changes of regional blood flow. At the same time, PEEP could reduce blood flow to liver and kidneys, glomerular filtration, urine output, and sodium excretion. ${ }^{8}$ The most important consequences of atelectasis include acute hypoxia, resistant hypoxia to increased oxygen concentration, increasing arterial alveolar oxygen gradient and reducing ventilation. ${ }^{9}$ Effective measures, in this case, include recruitment technique and use of PEEP. However, this method is not risk-free and cannot eliminate atelectasis. In the current work, we aimed to show effectiveness of PEEP during surgery on patient postoperative oxygen saturation and on prevention of atelectasis.

\section{Materials and methods}

The present study is a randomized double-blind clinical trial that was performed on patients undergoing elective cesarean section surgery. This study was approved by ethics committee of Birjand University of medical Sciences and registered in Iranian Registry of Clinical Trials (IRCT) number: IRCT2015072423315N1. The study was conducted on 120 individuals with ASA class I and II who were referred to Vali-Asr General Hospital in Birjand for cesarean section. The inclusion criteria for patients to be submitted to elective cesarean section were: BMI below 30, age 15 to 35 . Exclusion criteria included any chronic heart failure (CHF) related illness, pulmonary disease including asthma, allergy, emphysema, Chronic Obstructive Pulmonary Disease (COPD), bronchitis, pulmonary edema, aspiration, pulmonary embolism, pneumothorax, postoperative shivering, sepsis, pulmonary injury induced by blood transfusions, Acute Respiratory Distress Syndrome (ARDS), eclampsia and pre-eclampsia, or any incident during operation and ventilation that affects the arterial oxygen saturation. After the necessary explanation, the consent was obtained and the individual information questionnaire was completed. Patients enrolled were then randomly assigned to one of the three groups (A-B-C) according to the randomized blocking method.

On the operation bed, after the anesthetic induction, the hemodynamic indices including mean atrial pressure (MAP) and heart rate (HR) and arterial oxygen saturation $\left(\mathrm{SPO}_{2}\right)$ were recorded by a monitor, which was continued during operation and recorded every 15 minutes. The patient was then oxygenated with $100 \%$ of oxygen for three minutes. After induction of anesthesia, Thiopental injected at $5 \mathrm{mg} / \mathrm{kg}$ and succinylcholine injected at 1.5 $\mathrm{mg} / \mathrm{kg}$ and then a technician intubated the patient in less than 15 seconds.

Table 2. Comparison of MAP before, during and after surgery in patients in the three groups

\begin{tabular}{lllll} 
& \multicolumn{3}{c}{ Mean \pm SD } & \\
Groups & Pre-Operation & During-Operation & Post-Operation & In-p-value \\
A & $94 / 98 \pm 10 / 54$ & $94 / 08 \pm 12 / 90$ & $92 / 25 \pm 12 / 99$ & $0 / 49$ \\
B & $96 / 75 \pm 9 / 42$ & $93 / 18 \pm 8 / 98$ & $89 / 43 \pm 19 / 51$ & $<0 / 001$ \\
C & $95 / 83 \pm 12 / 70$ & $90 / 20 \pm 13 / 14$ & $90 / 5313 / 39$ & $0 / 005$ \\
p-value & $0 / 77$ & $0 / 31$ & $0 / 59$ & -
\end{tabular}


Positive end-expiratory pressure and arterial oxygen saturation after surgery

Eur J Transl Myol 28 (1): 93-97, 2018

Table 3. Comparison of mean heart rate before, during and after surgery in patients in the three groups Mean \pm SD

\begin{tabular}{llllc}
\cline { 2 - 3 } Groups & Pre-Operation & During-Operation & Post-Operation & In-p-value \\
A & $99 / 28 \pm 15 / 06$ & $96 / 98 \pm 16 / 69$ & $95635 \pm 10 / 76$ & $0 / 32$ \\
B & $103 / 70 \pm 15 / 81$ & $97 / 18 \pm 10 / 67$ & $98 / 98 \pm 10 / 45$ & $0 / 01$ \\
C & $96 / 38 \pm 14 / 73$ & $93 / 15 \pm 9 / 29$ & $95 / 30 \pm 9 / 81$ & $0 / 23$ \\
p-value & $0 / 10$ & $0 / 28$ & $0 / 22$ & -
\end{tabular}

Patients in group A underwent mechanical ventilation without PEEP. In group B, patients underwent ventilation with $5 \mathrm{~cm} \mathrm{H}_{2} \mathrm{O}$ PEEP and $10 \mathrm{~cm} \mathrm{H}_{2} \mathrm{O}$ under ventilation with PEEP in the group C. All patients received $50 \% \mathrm{~N}_{2} \mathrm{O}$ and $50 \%$ oxygen at the time of anesthesia. Anesthesia was maintained with Propofol at a dose of 100 $\mathrm{mg} / \mathrm{kg} / \mathrm{min}$ and $50 \% \mathrm{~N}_{2} \mathrm{O}$. After delivery, all patients received $100 \mu \mathrm{g}$ (microgram) of fentanyl, 40 units of oxytocin: 30 unit's intra-serum and ten units intravenously. During operation, in all patients, serum was ringer lactate. After the spontaneous respiratory depression, atracurium was injected at a dose of 0.2 $\mathrm{mg} / \mathrm{kg}$. At the end of the operation, and after the spontaneous respiration recovery, subjects were treated with neuromuscular blockers (Neostigmine $40 \mathrm{mg} / \mathrm{kg}$ and Atropine $20 \mathrm{mg} / \mathrm{kg}$ ). Statistical analysis was done with IBM SPSS version 24; one-way ANOVA, one-way ANOVA repeated measures and Kolmogorov-Simonov with the significant $P$ value $<0.05$. With $G^{*}$ Power software, the power of the analysis was determined to be higher than 80 percent.

\section{Results}

120 patients with caesarian section ASA (I and II) and anesthesia were enrolled in this study. Analyses showed no significant difference between the three groups A, B, and $\mathrm{C}$ (Table 1). Analysis failed to show any significant difference in $\mathrm{SPO}_{2}$ pre-, post-, and during operation among the three groups; however, post Tukey's analysis showed significant difference between groups $\mathrm{C}$ and $\mathrm{B}$ in comparison to group A (Table 2). Heart rates and MAP did not have any significant difference between the three groups A-B-C in pre-, post-, and during operation (Tables 3 and 4). However, analysis of MAP, heart rate, and mean of $\mathrm{SPO}_{2}$ during, pre- and post-operation showed a significant difference between each groups separately. According to the table 2, the results of one-way ANOVA showed that the mean arterial blood pressure before, during and after operation in patients in the three groups was not significantly different ( $p>0.05)$. The result of the in-group analysis showed that the average mean arterial pressure in patients in group A was not significantly different at the various stages (before, during and after operation) $(\mathrm{p}=0.49)$, but in patients with $\mathrm{B}$ and $\mathrm{C}$, a significant difference was achieved $(p<0.05)$. The result of Bonferroni's test showed that the mean arterial pressure in patients with $B$ and $C$ during and after surgery was significantly lower than before the operation ( $p$ $<0.05)$. Also, in group B, after surgery, there was a significant decrease in the time of operation $(p<0.05)$. According to the table 3 , the results of one-way ANOVA showed that the mean heart rate before, during and after operation in patients in the three groups did not differ significantly $(p>0.05)$. The results of in-group analysis of variance showed that the mean heart rate in patients in group $\mathrm{A}$ and $\mathrm{C}$ were not significantly different at the various stages (before, during and after the operation) ( $\mathrm{p}>$ $0.05)$, but there was a significant difference in group $B$ $(01 / 0=p)$. The results of Bonferroni's chi-square test showed that the mean heart rate in group B patients significantly decreased during and after surgery ( $\mathrm{p}$ $<0.05$ ). According to Table 4, the results of KruskalWallis test showed that the mean pre-operative arterial oxygen saturation did not differ significantly between the three groups $(\mathrm{p}=0.49)$, but during and after the operation, the significant difference was observed between these three groups ( $p<0.001$ ). The result of the Mann-Whitney test indicated that the mean of oxygen saturation of arterial blood oxygen during and after surgery with patients in group B and C was significantly higher than group A $(p<0.05)$. The result of Friedman test showed that the mean of oxygen saturation of arterial blood oxygen was significantly different in the patients in the three groups $\mathrm{A}, \mathrm{B}$, and $\mathrm{C}$ at the various stages (preoperative, during and after the operation) $(\mathrm{p}<0.001)$. The results of Wilcoxon test showed that the mean of oxygen saturation of arterial oxygen in patients of group A significantly increased during operation compared to the previous one and significantly decreased after surgery ( $p<0.05$ ). Also, the mean oxygen saturation of arterial oxygen in patients with $\mathrm{B}$ and $\mathrm{C}$ during and after the operation was significantly increased and significantly decreased after surgery $(\mathrm{p}<0.05)$.

\section{Discussion}

In this study we showed that using PEEP of 5 and $10 \mathrm{~cm}$ of $\mathrm{H}_{2} \mathrm{O}$ during the operation the arterial oxygen saturation was positively affected. Toyama et al. in 2012 investigated the effect of end-stage positive exertion on FRC (residual functional capacity of the lung) in low- 
Table 4. Comparison of mean oxygen saturation of arterial blood oxygen before, during and after operation in the three groups

\begin{tabular}{lllll} 
& \multicolumn{3}{c}{ Mean \pm SD } & \\
Groups & Pre-Operation & During-Operation & Post-Operation & In-p-value \\
A & $97 / 25 \pm 1 / 03$ & $98 / 25 \pm 1 / 13$ & $97 / 13 \pm 1 / 16$ & $<0 / 001$ \\
B & $98 / 30 \pm 0 / 94$ & $98 / 95 \pm 0 / 68$ & $98 / 98 \pm 10 / 45$ & $0 / 01$ \\
C & $96 / 95 \pm 1 / 04$ & $99 / 51 \pm 9 / 29$ & $98 / 50 \pm 0 / 91$ & $0 / 23$ \\
p-value & $0 / 49$ & $<0 / 001$ & $<0 / 001$ & -
\end{tabular}

volume ventilation during general anesthetic on nine patients with upper abdominal surgery. ${ }^{10}$ Patients were ventilated with face mask and $100 \%$ oxygen and PEEP $=$ $2 \mathrm{~cm} \mathrm{H}_{2} \mathrm{O}$ before anesthesia and FRC were measured. After tracheal intubation, the PEEP of 5 and 10 were recorded for 2 hours ( $7 \mathrm{ml} / \mathrm{kg}$ body weight). It was shown that FRC decreases by induction of anesthesia. Using a PEEP of about 5 to 10 centimeters of water can increase the lung functional capacity to the extent that the FRC is similar to that of a person's awakening. An one-blind clinical trial conducted in 2007 by Abedinzadeh et al., ${ }^{11}$ investigated the rate of reduction of arterial oxygen saturation after induction of anesthesia in 66 patients with three methods of peroxidation. In the first group, anesthesia was induced after the normal breathing using air oxygen. The second group consisted of $50 \%$ oxygen and $50 \%$ nitrous oxide, and the third group with $100 \%$ oxygen for 3 minutes, pre-oxygenated. After anesthetic induction, patients remained in apnea until $91 \%$ of their blood oxygen saturation, and then the time to reduce oxygen saturation is $91 \%$ for each patient. It was shown that pre oxygenation of patients for three minutes before surgery with normal breathing and $100 \%$ oxygen could significantly increase the duration of oxygen saturation to $91 \%$ in patients. A clinical trial study in 2013 by Golparvar et al., ${ }^{3}$ compared the effects of different levels of PEEP on hemodynamic and respiratory factors in patients with the healthy and damaged lung. During the clinical trial, patients were divided into two groups of 24 patients with healthy lungs and 28 patients with damaged lung. Then, different levels of PEEP were applied for 10 minutes. At first and at the end of each 10 minutes, the respiratory and hemodynamic characteristics of the patients were recorded and shown the increasing the level of the PEEP from 0 to 15 increased SPO2, decreased heart rate and blood pressure systolic and diastolic $(\mathrm{p}$ $<0.001) .^{2}$ Also, in the other study, the effect of PEEP on respiratory function in open heart surgery patients showed that arterial oxygen pressure was significantly higher in the two groups that found CPAP and IMV than the control group. The effect of mechanical ventilation with PEEP on the fate of pharmacokinetics of drugs has also been studied. In Najafi's study, the study of the pharmacokinetics of aminophylline following mechanical ventilation with PEEP showed that the volume of distribution and clearing of drugs in mechanical ventilation with positive pressure decreases. ${ }^{12,13}$ Therefore, if the positive pressure of the expiratory end is given at the right time, it can have significant effects on the respiratory function as well as the fate of the medications in the body. Dizon Satoh et al. used 0.5 and $10 \mathrm{~cm}$ water PEEP in 3 groups of patients with abdominal surgery, it was found that PEEP for 2 hours in patients undergoing abdominal surgery improved lung function and FRC. ${ }^{10,14,15}$

Our study showed that heart rate and mean arterial pressure did not differ significantly between the three groups and in different stages (before, during, after surgery).

In conclusion, the use of PEEP up to $5 \mathrm{~cm}$ of water during surgery could improve the hemodynamic state of the patients, in particular, the oxygenation of blood.

\section{List of acronyms}

AOS - arterial oxygen saturation

ARDS - Acute Respiratory Distress Syndrome

ASA - American Society of Anesthesiologists

BMI - body mass index,

CHF - chronic Heart Failure

COPD - Chronic Obstructive Pulmonary Disease

FRC - functional remaining capacity

HR - heart rate

IRCT - Iranian Registry of Clinical Trials

MAP - mean atrial pressure

PEEP - Positive end-expiratory pressure

$\mathrm{SPO}_{2}$ - arterial oxygen saturation

\section{Author's contributions}

Each author contributed in equal part to the manuscript.

\section{Acknowledgments and Funding}

We thank Dr. Zangoi and Ms. Adel who helped us in this study. This research received no specific grant from any funding agency in the public, commercial or not-forprofit sectors. 


\section{Positive end-expiratory pressure and arterial oxygen saturation after surgery}

Eur J Transl Myol 28 (1): 93-97, 2018

\section{Conflict of Interest}

The authors declare no conflicts of interests derived from the outcomes of this stud.

\section{Ethical Publication Statement}

We confirm that we have read the Journal's position on issues involved in ethical publication and affirm that this report is consistent with those guidelines.

\section{Corresponding Author}

Amir Sabertanha, Department of Anesthesia, Emam Reza General Hospital, Birjand University of Medical Sciences, Birjand, Iran.

E-mail: amirsaber43@yahoo.com

\section{E-mails of co-author}

Mahmood Ganjifard: ganji50@ hotmail.com

Masoumeh Samii: samiim@gmail.com

Samaneh Kouzegaran: kouzegarans@gmail.com

\section{References}

1. Pelosi P, Ravagnan I, Giurati G, et al. Positive endexpiratory pressure improves respiratory function in obese but not in normal subjects during anesthesia and paralysis. Anesthesiology 1999;91:1221-31.

2. Neumann P, Rothen H, Berglund J, et al. Positive end-expiratory pressure prevents atelectasis during general anaesthesia even in the presence of a high inspired oxygen concentration. Acta Anaesthesiol Scand 1999;43:295-301.

3. Golparvar M, Abbasi S, Jazi SK. The Effects of Different Levels of Positive End-Expiratory Pressure on Hemodynamic and Respiratory Indexes in Patients with Healthy and Damaged Lungs. Journal of Isfahan Medical School 2013;31(239).

4. Gander S, Frascarolo P, Suter M, et al. Positive endexpiratory pressure during induction of general anesthesia increases duration of nonhypoxic apnea in morbidly obese patients. Anesth Analg 2005;100:580-4.

5. Jardin F, Farcot J-C, Boisante L, et al. Influence of positive end-expiratory pressure on left ventricular performance. New England Journal of Medicine 1981;304:387-92.

6. Webb HH, Tierney DF. Experimental Pulmonary Edema due to Intermittent Positive Pressure Ventilation with High Inflation Pressures. Protection by Positive End-Expiratory Pressure. Am Rev Respir Dis. 1974;110:556-65.
7. Suter PM, Fairley HB, Isenberg MD. Effect of tidal volume and positive end-expiratory pressure on compliance during mechanical ventilation. Chest 1978;73:158-62.

8. Villar J, Kacmarek RM, Pérez-Méndez L, AguirreJaime A. A high positive end-expiratory pressure, low tidal volume ventilatory strategy improves outcome in persistent acute respiratory distress syndrome: a randomized, controlled trial. Crit Care Med 2006;34:1311-8.

9. Pelosi P, Caironi P, Bottino N, Gattinoni L. Positive end expiratory pressure in anesthesia. Minerva Anestesiol 2000;66:875-82. Review. Italian.

10. Satoh D, Kurosawa S, Kirino W, et al. Impact of changes of positive end-expiratory pressure on functional residual capacity at low tidal volume ventilation during general anesthesia. J Anesth 2012;26:664-9. doi: 10.1007/s00540-012-1411-9. Epub 2012 May 15.

11. Abedinzadeh M, Afzali L. The speed of decreasing of arterial oxygen saturation following induction of anesthesia, using 3 methods of pre-oxygenation techniques. Shahrekord University of Medical Scienes Journal 2007;9:10-5.

12. Najafi A, Shariat Mohairen R, Mojtahedzadeh M, et al. Tine: The effect of Positive End Expiratory Pressure on pharmacokinetic behavior of Aminophylline in patients with acute lung injury. Journal of Iranian Socety Anaesthesiology and Intensive Care 2003;23:26-34.

13. Fajardo MF, Claure N, Swaminathan S, et al. Effect of positive end-expiratory pressure on ductal shunting and systemic blood flow in preterm infants with patent ductus arteriosus. Neonatology 2014;105:9-13. doi: 10.1159/000355146. Epub 2013 Nov 1.

14. Auler Jr J, Carmona M, Barbas C, et al. The effects of positive end-expiratory pressure on respiratory system mechanics and hemodynamics in postoperative cardiac surgery patients. Braz J Med Biol Res 2000;33:31-42.

15. Gattinoni L, Caironi P, Cressoni M, et al. Lung recruitment in patients with the acute respiratory distress syndrome. N Engl J Med 2006;354:177586.

Received for publication: November 14, 2017 Accepted for publication: December 12, 2017 